of these known checkpoint inhibitors (PD1, CD152) could be important for SLE immunopathogenesis.

Secondly, the innate lymphoid cell 2 (ILC2) subset $\left(\mathrm{Lin}^{-} \mathrm{CD} 7^{+} \mathrm{CD} 25^{+} \mathrm{CD} 127^{+} \mathrm{G}\right.$ $\left.\mathrm{ATA}^{+}\right)$was markedly depressed in SLE $(0.11 \%, 0.1-0.255 \%)$ versus control $(0.41 \%, 0.25-0.55 \% ; p=0.0293)$. ILC2s protect epithelial integrity; a reduction suggests impaired protective roles in SLE.

Supervised cell frequencies from bivariate analysis correlate strongly with unsupervised cell frequencies, validating these results (Pearson's correlation coef-

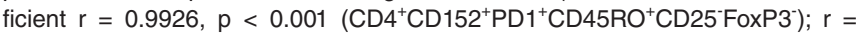
$0.8863, p<0.05$ (ILC2)).

Conclusion: With a multi-parametric, unbiased approach comparing SLE subjects to a large database of age-matched healthy controls, we identified two immune subsets of potential immunopathogenic importance. With this information, the CyTOF panel can be redesigned to probe more specifically into the SLE immunome, facilitating disease-specific interrogation.

References:

[1] Tsokos,G.C. (2011). Systemic lupus erythematosus. N Engl J Med 365(22), 2110-2121.

[2] Bombardier, C., Gladman, D.D., Urowitz, M.B., Caron, D., Chang, C.H. (1992). Derivation of the SLEDAI. A disease activity index for lupus patients. The Committee on Prognosis Studies in SLE. Arthritis Rheum 35(6), 630-640.

Disclosure of Interests: : None declared

DOI: 10.1136/annrheumdis-2020-eular.2855

\section{THU0238 ASSOCIATION OF SYSTEMIC DISEASES OF CONNECTIVE TISSUE (SDCT) AND GERONTOLOGICAL PROCESSES}

V. Shishkin ${ }^{1,2}$, G. V. Kudriavtseva ${ }^{2}$, Y. Malenkov ${ }^{2}$, V. V. Shishkin ${ }^{2} .{ }^{1}$ Saint Petersburg State University, Sankt-Peterburg, Russian Federation; ${ }^{2}$ Saint Petersburg State University, Sankt-Peterburg, Russian Federation

Background: The approach from the point of view of the evolutionary perspective of finding targets for therapeutic effects among the components of the cellular destruction system is critical both in the treatment of rheumatological diseases and in gerontology.

Objectives: To identify the relationships between the pathways of cell death in synovial fluid (SF) of people of different age groups with SDCT.

Methods: : SF was analyzed in patients of two age groups. Group N 1 of patients: 10 SLE ( $43 \pm 2.3$ years), 13 RA ( $45 \pm 1.6$ years), 7 SSD ( $35 \pm 1.8$ years) and 8 donors $(42 \pm 2.7$ years, postmortem). Group N 2 (age) of patients: 9 SLE $(69 \pm 1.8$ years), 10 RA ( $65 \pm 1.6$ years), 5 SSD $(65 \pm 0.7$ years) and 9 donors $(66 \pm 2.3$ years, postmortem). SF treated with $0.1 \%$ Triton- $\mathrm{x}-100$, resuspended in $0.1 \%$ citrate buffer $(\mathrm{pH}=7.4)$ and centrifuged at $25000 \mathrm{~g}$ for 30 minutes. The supernatant has been used in the experiment. The activity of adenosine monophosphate-activated protein kinase (AMPK) was evaluated by Western blotting. The level of p53 protein was analyzed by the enzyme immunoassay method using ELISA kit (eBioscience, (USA). The content of 8-hydroxy-2-deoxyguanosine (8-OH-dG) was evaluated using the EIA Kit (USA). Quantitative determination of cytochrome C (Cyt c) was carried using the ELISA kit. Active forms of oxygen free radicals (AFRF) were registered by EPR.

Results: : An interaction was established between the pathways of cell death in SDCT (mostly distinctive in SLE), age-related changes and clinical manifestations of the autoimmune process were established. The severity of cell death types depends on the nosological form of SDCT (tab.1).

TAB.1. SOME MARKERS OF AUTOPHAGY, APOPTOSIS AND NECROSIS IN SF OF DONORS AND PATIENTS WITH SDCT (M $\pm \mathrm{m})$ : AMPK (cond.unit/ $\mathrm{mg}$ protein), ATP-ase (nM Pi /min॰mg protein), AFRF (unit/mg protein); Cyt c, p 53, 8-OH-dG - (ng/ml)

\begin{tabular}{|c|c|c|c|c|c|c|}
\hline Group N1 & AMPK & ATP-ase & AFRF & Cyt c & p 53 & 8-OH-dG \\
\hline Donor (8) & $1,3 \pm 0,2$ & $3,7 \pm 0,3$ & $9,7 \pm 2,3$ & $10,4 \pm 0,3$ & $0,7 \pm 0,1$ & $9,5 \pm 0,8$ \\
\hline SLE (10) & $9,4 \pm 0,3^{* \star *}$ & $10,3 \pm 0,3^{*}$ & $44,9 \pm 3,6^{\star \star}$ & $40,1 \pm 2,9^{*}$ & $1,7 \pm 0,1^{*}$ & $28,9 \pm 1,4^{\star \star}$ \\
\hline$R A(13)$ & $7,3 \pm 0,2^{\star \star \star}$ & $9,7 \pm 0,5^{*}$ & $32,8 \pm 4,6^{\star \star}$ & $29,3 \pm 3,7^{*}$ & $1,5 \pm 0,06^{*}$ & $23,8 \pm 0,9^{*}$ \\
\hline SSD (7) & $4,3 \pm 0,2^{*}$ & $5,9 \pm 0,4$ & $17,8+4,3^{\star}$ & $18,9 \pm 3,7^{\star}$ & $0,9 \pm 0,1$ & $23,7 \pm 0,8^{*}$ \\
\hline Group N2 & AMPK & ATP-ase & AFRF & Cyt c & p 53 & 8-OH-dG \\
\hline Donor (9) & $3,7 \pm 0,4$ & $0,9 \pm 0,2$ & $24,8 \pm 5,1$ & $15,2 \pm 3,4$ & $1,3 \pm 0,2$ & $18,3 \pm 0,2$ \\
\hline SLE (9) & $18,2 \pm 0,2^{\star \star \star}$ & $4,2 \pm 0,3^{\star \star}$ & $64,6 \pm 3,1^{* *}$ & $52,8 \pm 3,9^{\star}$ & $3,4 \pm 0,1^{\star}$ & $44,8 \pm 0,7^{\star}$ \\
\hline$R A(10)$ & $17,0 \pm 0,4^{\star \star \star}$ & $4,0 \pm 0,2^{\star \star}$ & $54,9 \pm 1,6^{*}$ & $44,3 \pm 3,3^{*}$ & $3,2 \pm 0,3^{\star}$ & $42,2 \pm 0,4^{*}$ \\
\hline SSD (5) & $9,8 \pm 0,3^{\star *}$ & $2,8 \pm 0,2^{*}$ & $38,8 \pm 4,4^{\star}$ & $38,6 \pm 2,9^{*}$ & $2,9 \pm 0,1^{*}$ & $30,7 \pm 0,3^{*}$ \\
\hline
\end{tabular}

Notes: difference with the control norm: ${ }^{*}-p<0.05$; $^{* *}-p<0.01$; ${ }^{* \star}$ - $p<0.001$

Autophagy (especially in the case of SLE, as well as RA) is directly involved in the formation of the immune response and the inflammatory process. During aging, as well as during SDCT, there is a sharp increase in the activity of AMPK (intracellular energy sensor), expression of apoptosis inductors increases and also the acid-base balance shifts, the number of active forms of oxygen radicals increases, ox-red changes, the components of cellular destruction are activated, the activity of the cytokine system of the organism is disturbed (cytokines - regulators of apoptosis), the expression of chaperones decreases and immuno-oxygenase homeostasis shifts. Inhibition of the genetically determined process of cell death, apoptosis, underlies the development of autoimmune diseases, immunepro-liferative pathology (carcinogenesis) and gerontological changes.

Conclusion: The chaperone-mediated induction of the immune response as a signaling mechanism of autophagy, supposedly, is a common central link and a molecular switch that causes both the development of autoimmune diseases of connective tissue and geron-tological processes.

References:

[1] Shishkin V. I. et al. Ann Rheum Dis 2018; 77: sup.2 - A1274

Disclosure of Interests: None declared

DOI: 10.1136/annrheumdis-2020-eular.1154

\section{\begin{tabular}{|l|l}
\hline THU0239 DYNAMIC TEMPORAL CHANGES IN CLINICAL \\
\hline
\end{tabular} DISEASE ACTIVITY AND GUT MICROBIOTA REPRESENTATION OF A PATHOBIONT LINKED TO LUPUS NEPHRITIS}

G. Silverman ${ }^{1}$, D. Azzouz ${ }^{1}$, Z. Chen ${ }^{1}$, J. Deng $^{1}$, Z. Li ${ }^{2}$, D. Fenyo ${ }^{2}$,

A. Alekseyenko ${ }^{3} .{ }^{1} N Y U$ School of Medicine, Department of Medicine, New York, United States of America; ${ }^{2}$ NYU School of Medicine, Institute for Systems Genetics, New York, United States of America; ${ }^{3}$ MUSC, Biomedical Informatics Center, Charleston, United States of America

Background: From a cross-sectional cohort, we have identified a candidate human gut anaerobic pathobiont, Ruminococcus gnavus (RG) of the family Lachnospiraceae that was linked to active Lupus nephritis (LN)(1). Based on 16S rRNA amplicon analysis, LN patients displayed increased fecal RG abundance, concordant with serum IgG anti-RG antibody responses that appeared intertwined with anti-dsDNA responses implicated in renal pathogenesis. Indeed monocolonization of germ-free mice is reported to result in generalized inflammation and expansions of Th17 cells. However, RG at low levels are also prevalen in healthy adults, and the temporal dynamics of RG representation within Lupus microbiota ecosystems have not been investigated. Also, genomic sequences of few RG strains have been reported, and these vary greatly in genome structure, gene representation and sequence, which may have broad implications for adaptation to a host with systemic inflammation and/or factors that contribute to immune activation in a susceptible host

Objectives: To investigate the relationships between in vivo RG expansions and disease activity that often wax and wane overtime, we initiated longitudinal studies in Lupus patients and controls. As representation of $R G$ strains alone might alter pathogenic potential, we also sought to characterize RG strains from active LN patients. Methods: From our cohort, patients were characterized for demographics, clinical disease activity, and serologies including standard autoantibody and complement levels, and anti-bacterial responses of interest. High throughput 16S rRNA amplicon libraries from fecal samples were analyzed using QIIME 2 and DADA2 (1). Also, individual RG colonies were isolated and subjected to whole genome sequencing. Species and strains were then assigned in part based on multi-locus sequence typing and reference guided genomic assemblies.

Results: 16S rRNA analysis of 34 samples, at 2-4 timepoints from 14 SLE patients, documented highly conserved patterns of gut community representation overtime in 10/14 patients, based in part on unsupervised hierarchical cluster analysis. Notably, independent of vacillations in clinical disease activity of up to 8 SLEDAI points, conserved microbiome phylogenetic abundance/composition was documented at a family level, and the level of amplicon sequence variants that approximate identification of individual species. In pilot studies, from two active lupus nephritis patients hundreds of fecal bacterial colonies were isolated, with initial assignments by $16 \mathrm{~S}$ rRNA sequence. From highly redundant whole genome sequence analysis, these Lupus-patient fecal colonies were found to distribute into only four distinct $R G$ strains, which differed from reported strains.

Conclusion: Our findings suggest that many Lupus patients have little or no detectable perturbations in representation of the Lachnospiraceae family or abundance of RG species overtime. Moreover, this seeming microbiota stability was documented even in patients with dramatic changes in disease activity However, these approaches are inadequate to detect shifts between RG strains. In pilot studies we have isolated and characterized the genomes of four unique $R G$ strains from active LN patients, which include variations in gene conten and sequence that may have implications for the host-commensal relationship and immune activation. Broadening of these studies to larger number of SLE patients and healthy subjects, with metagenomic surveys of strain representation in genomic shotgun libraries are currently in progress, in coordination with 